\title{
Medical History Source
}

National Cancer Institute

\section{Source}

National Cancer Institute. Medical History Source. NCI Thesaurus. Code C156812.

The source providing the medical history. 